\title{
Upper and lower bounds for expected utility ${ }^{\star}$
}

\author{
Marco LiCalzi \\ Department of Applied Mathematics, University of Venice, 30123 Venezia, ITALY \\ (e-mail: licalzi@unive.it)
}

Received: December 14, 1999; revised version: March 8, 2000

Summary. The decision-theoretic literature has developed very few techniques to bound the expected utility of a random variable when only simple statistics like its median or mode or mean are known. One reason for this lack of results is that we are missing a convenient way to link probability theory and expected utility. This paper is written to demonstrate a general (and genuinely probabilistic) technique to obtain upper and lower bounds for the expected utility of a lottery.

Keywords and Phrases: Expected utility, Bounds, Dominance, Inequalities.

JEL Classification Numbers: D81, C44.

\section{Introduction}

The theoretical importance of the expected utility model in the study of behavior under risk is unquestioned. Its practical use, however, is hampered by many difficulties. One of these is that both the agent's utility function $U$ and the cumulative distribution function $F$ of the random variable $X$ must be available in order to compute $E U(X)$. In reality, one has often to settle for much less since either $U$ or $F$ may be imperfectly known.

This leads to the problem of estimating expected utilities when the relevant information is incomplete. For example, suppose that the agent is known to be risk averse but that her (increasing) utility function $U$ is not fully specified. If the expected value $E(X)$ of the random variable $X$ exists, Jensen's inequality implies that her expected utility $E U(X)$ for $X$ is bounded above by the utility of

\footnotetext{
* I am grateful to Yosi Rinott and to my former students Stefano DellaVigna and Francesca Nieddu for our conversations, as well as to the seminar audiences at the University of California (San Diego) and Paris VI for their comments.
} 
$E(X)$. This is sufficient to conclude that any sure outcome $x>E(X)$ is preferred to $X$.

More generally, however, the decision-theoretic literature has largely been silent on the problem of estimating bounds for the expected utility when only little information about either $X$ or $U$ is known. Although there is a significant area of probability theory specializing in techniques to establish inequalities, these have not been brought to bear on the problem.

To my knowledge, the most notable exceptions are the following. Vickson (1977) studies the case where the utility function $U(x)$ is known only at a number of points. Willassen (1981, respectively, 1990) applies the general theory of Tchebycheff bounds to derive upper and lower bounds for $E U(X)$ when the first two (respectively, three) moments of $X$ are known and the first three (respectively, four) derivatives of $U$ alternate in sign. Birge and Teboulle (1989) assumes convexity of $U$ and applies techniques based on the knowledge of the moments of $X$.

One reason for this shortage of results is that we are missing a convenient way to link probability theory and expected utility. The purpose of this paper is to demonstrate a general (and genuinely probabilistic) technique to derive upper and lower bounds for the expected utility of a random variable. This technique may accomodate different assumptions about the knowledge available on $U$ and $F$. For example, Theorem 12 below provides a lower bound for the expected utility of a risk averse agent; Theorems 3, 9, and 10, instead, deal with the $S$ shaped utility functions advocated by Kahneman and Tversky (1979)'s prospect theory.

To describe our technique, let us agree to write $X \sim F$ to denote that the random variable (r.v.) $X$ has a cumulative distribution function (c.d.f.) $F$. The basic idea is based on a probabilistic interpretation of the expected utility model advanced in Castagnoli and LiCalzi (1996). Assume that the utility function $U$ is bounded, increasing (but not constant) and continuous. By an appropriate positive affine transformation, normalize $U$ so that $\inf U(x)=0$ and $\sup U(x)=1$. Then $U$ satisfies all the properties characterizing a c.d.f., as was first noted in Berhold (1973).

Therefore, there exists (on some appropriate probability space) a random variable $V$ with cumulative distribution function $U$. Moreover, the r.v. $V \sim U$ can be taken to be stochastically independent of any given $X \sim F$. See Theorem 14.1 in Billingsley (1986). This implies that we can let $U(x)=P(V \leq x)$ and rewrite the expected utility of $X$ as

$$
E U(X)=\int U(x) \mathrm{d} F(x)=\int P(V \leq x) \mathrm{d} F(x)=P(X \geq V) .
$$

Hence, the problem to derive bounds on $E U(X)$ can be attacked by studying how to bound the probability that the r.v. $X \sim F$ is greater than another (stochastically independent) r.v. $V \sim U$.

It is worthwhile pausing a bit on the dual role of $U$. In the expected utility model, this is a (von Neumann and Morgenstern) utility function; in this paper, 
instead, we assume inf $U(x)=0$ and $\sup U(x)=1$ and exploit the fact that $U$ can be considered a cumulative distribution function. Bordley and LiCalzi (2000) and Castagnoli and LiCalzi (1996) argue at some length on the possible advantages of this second viewpoint in a decision-theoretic context. Here, however, all that is needed is that the equality displayed in Equation (1) holds true and therefore that bounding $P(X \geq V)$ is tantamount to bounding $E U(X)$.

The paper stresses this point in two ways. First, we write the statement of our results in terms of bounds on $E U(X)$ but provide proofs with reference to $P(X \geq V)$. Second, we let our discussion liberally switch between the first and the second interpretation of $U$ depending on the context.

\section{Preliminaries}

Unless explicitly noted, we assume throughout the paper that $U$ is continuous. This natural but slightly restrictive assumption can be axiomatically justified in the expected utility model. See Grandmont (1972). Its purpose here is to rule out some minor technicalities regarding the Riemann-Stieltjes integral involved in Equation (1). On the other hand, note that we make no assumptions on the c.d.f. $F$.

We also follow two simplifying conventions. First, we take the domain of the c.d.f. $F$ of a r.v. $X$ to be the set of extended reals $\mathbb{R}^{*}$. Hence, $F$ is positive, increasing, right-continuous and such that $F(-\infty)=0$ and $F(+\infty)=1$. Second, we consistently pair the r.v.'s $X, Y, Z, V$ with their respective c.d.f.'s $F, G, H, U$ so that $X \sim F, Y \sim G, Z \sim H$, and $V \sim U$ throughout the paper.

Our first result is a simple equality.

Theorem 1 Let $Z_{1}$ and $Z_{2}$ be two r.v.'s uniformly distributed on [0,1], respectively stochastically independent of $X$ and $V$. Then

$$
E U(X)=P\left(U(X) \geq Z_{1}\right)=P\left(Z_{2} \geq F(V)\right) .
$$

Proof. We prove only the first equality. By the monotonicity of $U, P(X \geq V)=$ $P(U(X) \geq U(V))$. Take $Z_{1} \stackrel{d}{=} U(V)$ and stochastically independent of $X$.

This result is not interesting, but it illustrates a point. When working on bounds for $P(X \geq V)$, one may often obtain different statements depending on whether $X$ or $V$ are considered. Hence, many of our results admit two distinct formulations which are however based essentially on the same proof. When both formulations are offered, we always omit the proof of the dual result.

\section{Cut-off inequalities}

Given $X \sim F$ and $Y \sim G$, we say that $X$ (first-order) stochastically dominates $Y$ and we write $X \geq_{s t} Y$ if and only if $F(x) \leq G(x)$ for all $x \in \mathbb{R}$. Our first result is also proved as Thm. 3.4 in Wrather and Yu (1982, p. 322). 
Theorem 2 Suppose that $X \geq_{s t} V$. Then $E U(X) \geq 1 / 2$.

Proof. By stochastic dominance, $U(x) \geq F(x)$ for all $x$. Hence,

$$
P(X \geq V)=\int U(x) \mathrm{d} F(x) \geq \int F(x) \mathrm{d} F(x)=1 / 2 .
$$

Recall that we assume $U$ bounded between 0 and 1 . Hence, the expected utility of an arbitrary r.v. $X$ is a number in $[0,1]$. Thus, the assumption that $U(x) \geq F(x)$ for all $x$ guarantees that $E U(X)$ achieves a value that is at least halfway in the interval $[0,1]$. This lower bound is sharp: just take a stochastically independent r.v. $X \stackrel{d}{=} V$ (i.e., $F=U$ ) and check that $E U(X)=1 / 2$.

This result may be extended into two directions. The first one restricts the family of random variables out of which $X$ and $V$ may be chosen; the second one allows for a finer ordering.

We say that a r.v. $X \sim F$ is symmetric if there exists some $m$ in $\mathbb{R}$ such that $F(m-x)+F(m+x)=1$ for almost all $x \in \mathbb{R}$. We call $m$ the symmetric median of $X$; in fact, when $F$ is continuous, taking limits for $x \rightarrow 0$ in the previous equality gives $F(m)=1 / 2$. Our definition implies that $m$ is unique even if the set of $x$ 's such that $F(x)=1 / 2$ is not a singleton. A version of the next result appears in DellaVigna (1997).

Theorem 3 Let $X$ and $V$ be symmetric r.v.'s respectively with symmetric medians $m_{X}$ and $m_{V}$. Suppose that at least one between $X$ and $V$ has an absolutely continuous c.d.f. Then

$$
m_{X} \geq m_{V} \quad \text { if and only if } \quad E U(X) \geq 1 / 2,
$$

with equality holding if $m_{X}=m_{V}$.

Proof. Since $X$ is symmetric, $\mathrm{d} F\left(m_{X}+y\right)+\mathrm{d} F\left(m_{X}-y\right)=0$. Hence,

$$
\begin{aligned}
P & (X \geq V)=\int_{-\infty}^{+\infty} U(x) \mathrm{d} F(x)=\int_{-\infty}^{m_{X}} U(x) \mathrm{d} F(x)+\int_{m_{X}}^{+\infty} U(x) \mathrm{d} F(x) \\
& =-\int_{0}^{+\infty} U\left(m_{X}-y\right) \mathrm{d} F\left(m_{X}-y\right)+\int_{0}^{+\infty} U\left(m_{X}+y\right) \mathrm{d} F\left(m_{X}+y\right) \\
& =\int_{0}^{+\infty}\left[U\left(m_{X}-y\right)+U\left(m_{X}+y\right)\right] \mathrm{d} F\left(m_{X}+y\right) .
\end{aligned}
$$

Consider the argument of the last integral. If $m_{X}=m_{V}$, the symmetry of $V$ implies $U\left(m_{X}-y\right)+U\left(m_{X}+y\right)=1$ a.e., with equality holding everywhere if $U$ is continuous. Hence, by the absolute continuity of $F$ or $U$,

$$
\begin{aligned}
P(X \geq V) & =\int_{0}^{+\infty}\left[U\left(m_{X}-y\right)+U\left(m_{X}+y\right)\right] \mathrm{d} F\left(m_{X}+y\right) \\
& =\int_{0}^{+\infty} \mathrm{d} F\left(m_{X}+y\right)=\frac{1}{2} .
\end{aligned}
$$

If instead $m_{X}-m_{V}=\varepsilon>0$, then 


$$
\begin{aligned}
U\left(m_{X}-y\right)+U\left(m_{X}+y\right) & =U\left(m_{V}+\varepsilon-y\right)+U\left(m_{V}+\varepsilon+y\right) \\
& \geq U\left(m_{V}-y\right)+U\left(m_{V}+y\right)=1
\end{aligned}
$$

and therefore

$$
\begin{aligned}
P(X \geq V) & =\int_{0}^{+\infty}\left[U\left(m_{X}-y\right)+U\left(m_{X}+y\right)\right] \mathrm{d} F\left(m_{X}+y\right) \\
& \geq \int_{0}^{+\infty} \mathrm{d} F\left(m_{X}+y\right)=\frac{1}{2} .
\end{aligned}
$$

For $m_{X}-m_{V}=\varepsilon<0$, reverse the above argument.

When $V$ is symmetric, its c.d.f. $U$ is $S$-shaped around the inflexion point $m_{V}$. In the language of expected utility, then, the assumptions of Theorem 3 imply a utility function $U$ consistent with the reflection effect described in Kahneman and Tversky (1979), provided that the agent sets her reference point to $m_{V}$.

Assuming this type of utility function and a choice set of symmetric r.v.'s, Theorem 3 implies that one may learn a lot just from comparing their (symmetric) medians: a (symmetric) r.v. $X$ with $m_{X} \geq m_{V}$ must have a higher expected utility than any other (symmetric) r.v. $Y$ with $m_{Y}<m_{V}$. This suggests a simple rule to prune the choice set of this kind of expected utility maximizer when she is choosing over symmetric r.v.'s: if there is an available lottery $X$ with $m_{X} \geq m_{V}$, ignore all r.v.'s with $m_{Y} \leq m_{V}$.

We call this rule search over (symmetric) medians. Searching over symmetric medians can be used to partition all r.v.'s into two classes: those with (symmetric) median above and respectively below $m_{V}$. Only r.v.'s in the first class are candidates to maximize the expected utility. Note that this rule cannot be strengthened to say that $m_{X}>m_{Y}$ implies $E U(X)>E U(Y)$ : for instance, if $X$ takes values 0 and 4 and $Y$ takes values $1 / 2$ and $3 / 2$ (both with equal probability), then $m_{x}=2>1=m_{Y}$; however, for $V$ uniformly distributed on [0,1], $E U(X)=1 / 2<3 / 4=E U(Y)$. The direct comparison of medians between r.v.'s in the same class is not conclusive.

A second extension of the cut-off inequality in Theorem 2 is inspired by ideas first presented in Karlin and Novikoff (1963). We say that a c.d.f. $F$ crosses from below another c.d.f. $U$ if there exists a point $c \in \mathbf{R}^{*}$ such that $F(x) \leq U(x)$ for all $x \leq c$ and $F(x) \geq U(x)$ for all $x>c$. Note that, using the right-continuity of $F$ and $U$, this implies $F(c) \geq U(c)$ and thus $F(c)=U(c)$. Also, note that $c$ is an extended real. When $F$ crosses $U$ from below in $c=+\infty$, then $X \geq_{s t} V$. A version of the next result appears in Nieddu (1997).

Theorem 4 Let $X \sim F$ and $V \sim U$ be such that $F$ crosses $U$ from below in $c$. Then

$$
F(c)-\frac{F^{2}(c)}{2} \leq E U(X) \leq \frac{1+F^{2}(c)}{2} .
$$

Similarly, if $U$ crosses $F$ from below, then

$$
\frac{1-F^{2}(c)}{2} \leq E U(X) \leq 1-F(c)+\frac{F^{2}(c)}{2} .
$$


Proof. We prove only the upper bound of the first set of inequalities.

$$
\begin{aligned}
P(X \geq V) & =\int_{-\infty}^{c} U(x) \mathrm{d} F(x)+\int_{c}^{+\infty} U(x) \mathrm{d} F(x) \\
& \leq U(c) F(c)+\int_{c}^{+\infty} F(x) \mathrm{d} F(x)=\frac{1+F^{2}(c)}{2} .
\end{aligned}
$$

The other bounds follow from similar arguments.

Differently from the previous ones, this result provides both upper and lower (non trivial) bounds. It is then interesting to consider the length of the interval of possible values for $E U(X)$. Taking the difference between upper and lower bound in Equation (2), we find $1 / 2-F(c)+F^{2}(c)$. This value is minimized for $F(c)=1 / 2$; that is, when $F$ crosses $U$ from below (or from above) at the median of $X$. In this case, Equation (2) provides the tight bound $3 / 8 \leq E U(X) \leq 5 / 8$. Analogous conclusion holds for Equation (3).

The bounds are sharp. For instance, assume that $V$ is uniformly distributed on $[0,1]$ so that $U(x)=\min \{1, \max \{0, x\}\}$. If the c.d.f. of $X$ is $F(x)=0$ for $x<1 / 2$ and $F(x)=\min \{x, 1\}$ for $x \geq 1 / 2$ so that $F$ crosses $U$ from below at the median of $X$, then $E U(x)=5 / 8$. Finally, note that when $F$ crosses $U$ from below in $c=+\infty$, Theorem 4 implies Theorem 2 .

\section{Quantile-based inequalities}

We say that a point $x$ in $\mathbf{R}$ is of strict increase for a c.d.f. $F$ if $F(x-\varepsilon)<F(x+\varepsilon)$ for all $\varepsilon>0$. The support of $F$ is the set of its points of strict increase and will be denoted by $S_{F}$. Note that $S_{F}$ is a closed subset of $\mathbb{R}$; moreover, $F$ is strictly increasing (and hence invertible) on its support if and only if $S_{F}$ is an interval.

The generalized inverse function of a c.d.f. $F$ is

$$
F^{(-1)}(z)=\inf \left\{x \in \mathbf{R}^{*}: F(x) \geq z\right\}
$$

The generalized inverse $F^{(-1)}:[0,1] \rightarrow \mathbf{R}^{*}$ is positive, increasing, leftcontinuous and such that $F^{(-1)}(0)=-\infty$ and $F^{(-1)}(1)=\sup S_{F}$. Moreover, for any point $x$ in the support of $F, F^{(-1)} \circ F(x)=x$; in particular, if $S_{F}$ is an interval, $F^{(-1)}=F^{-1}$ on $F\left(S_{F}\right)$.

Let $X \sim F$. Given $\alpha \in[0,1]$, we say that $x_{\alpha}=F^{(-1)}(\alpha)$ is the $\alpha$-quantile of $F$. The $\alpha$-quantile exists and is unique for any $\alpha \in[0,1]$. Given $0=\alpha_{0}<$ $\alpha_{1}<\alpha_{2}<\ldots<\alpha_{n}<\alpha_{n+1}=1$, we denote the corresponding quantiles of $F$ by $x_{(0)}, x_{(1)}, \ldots, x_{(n)}, x_{(n+1)}$. Note that $x_{(0)}=-\infty$ and $x_{(n+1)}=\sup S_{F}$.

The results in this section concern the case where only the values of a few quantiles are known for either $U$ or $F$. Since in applications it is usually the case that there is less information about $U$ than about $F$, we cast our presentation in terms of the quantiles $v_{(0)}, v_{(1)}, \ldots, v_{(n)}, v_{(n+1)}$ of $U$. 
Theorem 5 Suppose that the support of $U$ is an interval $[a,+\infty)$. Given any r.v. $X \sim F$,

$$
\alpha_{n}-\sum_{k=1}^{n} F\left(v_{k}\right)\left[\alpha_{k}-\alpha_{k-1}\right] \leq E U(X) \leq 1-\sum_{k=1}^{n} F\left(v_{k}\right)\left[\alpha_{k+1}-\alpha_{k}\right] .
$$

Proof. Breaking up the domain of integration,

$$
P(X \geq V)=\int_{-\infty}^{+\infty} P(V \leq x) \mathrm{d} F(x)=\sum_{k=0}^{n} \int_{v_{(k)}}^{v_{(k+1)}} P(V \leq x) \mathrm{d} F(x) .
$$

For each term in the sum, consider the inequalities

$$
\begin{aligned}
\alpha_{k}\left[F\left(v_{(k+1)}\right)-F\left(v_{(k)}\right)\right] \leq \int_{v_{(k)}}^{v_{(k+1)}} & P(V \leq x) \mathrm{d} F(x) \leq \\
& \leq U\left(v_{(k+1)}\right)\left[F\left(v_{(k+1)}\right)-F\left(v_{(k)}\right)\right] .
\end{aligned}
$$

Since $U$ is invertible on $U([a,+\infty)), U\left(v_{(k+1)}\right)=\alpha_{k+1}$. Hence, substituting Equation (6) into Equation (5), we obtain

$$
\begin{aligned}
\sum_{k=0}^{n} \alpha_{k}\left[F\left(v_{(k+1)}\right)-F\left(v_{(k)}\right)\right] \leq P(V & \leq X) \leq \\
& \leq \sum_{k=0}^{n} \alpha_{k+1}\left[F\left(v_{(k+1)}\right)-F\left(v_{(k)}\right)\right] .
\end{aligned}
$$

Noting that $F\left(\sup S_{U}\right)=F(+\infty)=1$, the result follows by rearranging the sums.

The applicability of this result is immediate. After a once-and-for-all estimate of a few $\alpha$-quantiles of $U$, bounding the expected utility of a r.v. $X \sim F$ requires only to compute the values of its c.d.f. at these quantiles. Theorem 5 may also be easily generalized to accomodate the assumption that $\sup S_{U}<+\infty$ or that the support of $U$ is not an interval. However, we think it more effective to maintain here these two mild restrictions and pause on the special case where only the median of $U$ is known.

For $\alpha=1 / 2$, the $\alpha$-quantile of $F$ is called the median of $X$, that we keep denoting by $m_{X}$. Note that the symmetric median of a symmetric r.v. $X \sim F$ (as defined just before Theorem 3) always coincides with its median $m$, except for the very special case where $F(m)=1 / 2$ and $m$ is not a point of strict increase for $F$ : then it is strictly greater than its median.

Corollary 6 Suppose that the support of $U$ is an interval $[a,+\infty)$ and let its median be $m$. Given any r.v. $X \sim F$,

$$
\frac{1-F(m)}{2} \leq E U(X) \leq \frac{2-F(m)}{2} .
$$


Proof. First, note that the proof of Theorem 5 still goes through when $\sup S_{U}=$ $+\infty$. Then substitute $n=1$ with $\alpha_{1}=1 / 2$ in Equation (4).

The bounds in Corollary 6 are (asymptotically) sharp. For instance, take the exponential utility function $U(x)=1-e^{-x}$ and assume that any r.v. $X_{k}$ in the choice set is exponentially distributed with parameter $k>0$. Then the median of $U$ is $m=\log 2$ and the expected utility of $X_{k}$ is $E U\left(X_{k}\right)=1 /(1+k)$. Corollary 6 provides the bounds

$$
\frac{1}{2^{k+1}} \leq E U\left(X_{k}\right) \leq \frac{1+2^{k}}{2^{k+1}} .
$$

As $k \rightarrow 0$, both $E U\left(X_{k}\right)$ and the upper bound tend to 1 . Similarly, as $k \rightarrow+\infty$, both $E U\left(X_{k}\right)$ and the lower bound tend to 0 . Analogous results of (asymptotic) sharpness hold for the bounds in Theorem 5.

Corollary 6 leads to another result that is probably more interesting from a decision-theoretic point of view.

Corollary 7 Suppose that the support of $U$ is an interval $[a,+\infty)$. Let $m_{X}$ and $m_{V}$ be respectively the medians of $X$ and $V$.

$$
\begin{aligned}
& \text { i) If } m_{X}=m_{V} \text {, then } 1 / 4 \leq E U(X) \leq 3 / 4 \text {. } \\
& \text { ii) If } m_{X}>m_{V} \text {, then } E U(X) \geq 1 / 4 . \\
& \text { iii) If } m_{X}<m_{V} \text {, then } E U(X) \leq 3 / 4 \text {. }
\end{aligned}
$$

Proof. Note that $1 / 2=F\left(m_{X}\right) \geq F\left(m_{V}\right)$ if and only if $m_{X} \geq m_{V}$ and derive the results from the bounds in Equation (8).

When $U$ satisfies the assumptions of Corollary 7, this suggests another simple rule to prune the choice set of an expected utility maximizer choosing over arbitrary r.v.'s. If there exists a r.v. $X$ such that $E U(X) \geq 3 / 4$, ignore all r.v.'s y $Y$ with $m_{Y} \leq m_{V}$. We call this rule searching over medians.

The results in this section admit dual formulations when there is incomplete information about the c.d.f. $F$. For the sake of brevity, here we only state (and do not prove) the equivalent of Theorem 5. Given $X \sim F$ and $0=\alpha_{0}<\alpha_{1}<$ $\ldots<\alpha_{n}<\alpha_{n+1}=1$, denote the respective quantiles of $F$ by $x_{(0)}, x_{(1)}, \ldots, x_{(n+1)}$. Note that $U$ is not required to be continuous.

Theorem 8 Suppose that the r.v. $X$ has a continuous c.d.f. F whose support is an interval $[a,+\infty)$. Given any increasing, bounded (but possibly not continuous) utility function $U$,

$$
\sum_{k=1}^{n} U\left(x_{(k)}\right)\left[\alpha_{k+1}-\alpha_{k}\right] \leq E U(X) \leq \sum_{k=0}^{n} U\left(x_{(k+1)}\right)\left[\alpha_{k+1}-\alpha_{k}\right] .
$$

\section{Mode-based inequalities}

We say that a r.v. $X \sim F$ is unimodal if there exists a mode $M \in \mathbb{R}$ such that $F$ is convex on $(-\infty, M)$ and concave on $(M,+\infty)$. For simplicity, in the following 
we assume that the mode of $X$ is unique, but our results may be generalized to the case of multiple modes. Note that, except possibly in $x=M$, the c.d.f. $F$ of a unimodal r.v. $X$ is continuous.

When $V$ is unimodal, its c.d.f. $U$ is $S$-shaped around the inflexion point $m_{V}$. However, differently from the case of a symmetric $V$, the two arms of the $S$ may not have the same curvature. Hence, this kind of utility function is consistent not only with the reflection effect, but with a steeper slope over losses as well. This makes it a prominent candidate as value function for the prospect theory of Kahneman and Tversky (1979).

Theorem 9 Suppose that $V \sim U$ is unimodal with mode $M$. Let $U$ be absolutely continuous with density function $u$ and $\inf S_{U} \geq 0$. If the r.v. $X$ is positive and integrable, then

$$
E U(X) \leq u(M) E(X) .
$$

Proof. Since $M$ is the mode of $u, u(v) \leq u(M)$ for all $v$ in $S_{U}$. Hence,

$$
\begin{aligned}
P(X \geq V) & =\int_{S_{U}} P(X \geq v) u(v) \mathrm{d} v \leq u(M) \int_{S_{U}} P(X \geq v) \mathrm{d} v \\
& =u(M) \int_{S_{U}}(1-F(v)) \mathrm{d} v \leq u(M) E(X),
\end{aligned}
$$

where the second equality follows from the fact that the set on which $P(X \geq$ $v) \neq P(X>v)$ has Lebesgue measure zero. The last inequality exploits the well-known equality (see Shiryayev, 1984, Corollary II.6.2, p. 206)

$$
E(X)=\int_{0}^{+\infty}(1-F(x)) \mathrm{d} x
$$

controlling for $S_{U} \subseteq[0,+\infty)$.

Whenever $u(M)<1 / E(X)$, this simple inequality is not trivial. Let us consider an example. Suppose that both $X$ and $V$ are exponentially distributed with parameters respectively $\alpha>0$ and $\beta>0$. In particular, this implies $U(x)=\max \left\{0,1-e^{-\beta x}\right\}$. Then $E(X)=1 / \alpha$ while the mode of $V$ is $M=0$, so that $U(M)=\beta$. Theorem 9 states that

$$
E U(X)=\frac{\beta}{\alpha+\beta} \leq \frac{\beta}{\alpha}=u(M) E(X)
$$

and the upper bound is more and more binding as $\beta / \alpha \rightarrow 0$. In particular, note that $V \geq_{s t} X$ if and only if $\beta \leq \alpha$. Hence, by the dual to Theorem 2 , for $\beta<\alpha$ we might derive the upper bound $E U(X) \leq 1 / 2$. However, for $\alpha>2 \beta$, the mode-based upper bound given in (10) is tighter than this latter one based on stochastic dominance.

The upper bound in Theorem 9 is sharp. For instance, assume that $V$ is uniformly distributed on $[0,1]$ so that $u(M)=1$. Then, for any r.v. $X$ on $[0,1]$, $E U(X)=E(X)$ and (10) holds with equality. 
Theorem 9 gives an upper bound when the support of $U$ is bounded from below by 0 . The next result provides two different upper bounds for the more general case where $S_{U}$ is simply bounded from below.

Theorem 10 Suppose that $V \sim U$ is unimodal with mode $M$. Let $U$ be absolutely continuous with density function $u$ and $\inf S_{U}=a>-\infty$. Then both

$$
E U(X) \leq E u(X) \cdot(M-a)+P(X>M)
$$

and

$$
E U(X) \leq u(M) \cdot P(X \leq M) \cdot(M-a)+P(X>M) .
$$

Proof. We begin with the first inequality. For $v \leq M$,

$$
\begin{aligned}
u(v) P(X \geq v) & =u(v) \int_{x \geq v} \mathrm{~d} F(x) \\
& \leq \int_{v \leq x \leq M} u(x) \mathrm{d} F(x)+u(v) \int_{x>M} \mathrm{~d} F(x) \\
& \leq E u(X)+u(v) P(X>M)
\end{aligned}
$$

Hence,

$$
\begin{aligned}
P(X \geq V) & =\int_{a}^{M} u(v) P(X \geq v) \mathrm{d} v+\int_{M}^{b} u(v) P(X \geq v) \mathrm{d} v \\
& \leq \int_{a}^{M}[E u(X)+u(v) P(X>M)] \mathrm{d} v+\int_{M}^{b} u(v) P(X>M) \mathrm{d} v \\
& =E u(X)(M-a)+P(X>M) .
\end{aligned}
$$

The proof of the second inequality is similar. For $v \leq M$,

$$
\begin{aligned}
u(v) P(X \geq v) & =u(v) \int_{x \geq v} \mathrm{~d} F(x) \\
& \leq u(M) \int_{v \leq x \leq M} \mathrm{~d} F(x)+\int_{x>M} u(v) \mathrm{d} F(x) \\
& \leq u(M) P(X \leq M)+u(v) P(X>M) .
\end{aligned}
$$

The rest goes on as above.

Inequalities (11) and (12) provide different upper bounds for $E U(X)$. Comparing the two expressions on the right-hand side, it is obvious that which one is tighter depends on which one is smaller between $E u(X)$ and $u(M) P(X \leq M)$. The following example shows that both cases are possible.

Indeed, suppose that $V$ has a Beta c.d.f. with parameters $\alpha=\beta>1$. Then $V$ is symmetric around its mode $M=1 / 2$. If $X$ takes only values 0 and 1 with probability $1 / 2$, then 


$$
E u(X)=\frac{1}{2} u(0)+\frac{1}{2} u(1)=0<\frac{\Gamma(2 \alpha)}{2 \Gamma(\alpha)}\left(\frac{1}{2}\right)^{2 \alpha-1}=u(M) P(X \leq M) .
$$

This can be used to show that Inequality (11) is sharp. In fact, note that either Theorem 3 or simple symmetry considerations imply that $E U(X)=1 / 2$. Thus, substituting $E u(X)=0, M=1 / 2$ and $a=0$ in (11), we find

$$
E U(X)=\frac{1}{2}=E u(X)(M-a)+P(X>M) .
$$

On the other hand, consider the case where $X$ takes only the values $(1 / 2)-\varepsilon$ and $(1 / 2)+\varepsilon$ with probability $1 / 2$. Then

$$
u(M) P(X \leq M)=\frac{\Gamma(2 \alpha)}{2 \Gamma(\alpha)}\left(\frac{1}{2}\right)^{2 \alpha-1}<\frac{\Gamma(2 \alpha)}{2 \Gamma(\alpha)}\left(\frac{1}{4}-\varepsilon^{2}\right)^{\alpha-1}=E u(X)
$$

for sufficiently small $\varepsilon>0$ and $\alpha>1$. Again, either Theorem 3 or simple symmetry considerations imply $E U(X)=1 / 2$. Taking for instance $\alpha=3 / 2$, (12) gives the upper bound

$$
E U(X)=\frac{1}{2}<\frac{1}{4 \sqrt{\pi}}+\frac{1}{2}=u(M) P(X \leq M)(M-a)+P(X>M) .
$$

The bound offered by (12) is sharp. For instance, assume that $V$ is uniformly distributed on $[0,1]$ and that $X$ is degenerate on $x=1$. Then $E U(X)=1=$ $P(X \geq V)=1=u(M) \cdot P(X \leq M) \cdot(M-a)+P(X>M)$. However, we suspect (but have not been able to prove) that it is not sharp (even asymptotically) unless the support is a singleton.

\section{Markov-like inequalities}

Most of this section is based on the following generalization of Markov's inequality. See for instance Davidson (1994, Corollary 9.11, p. 132).

Lemma 11 Let $g: \mathbb{R} \rightarrow \mathbb{R}$ be a bounded function. Suppose that, for a given constant $a, x \geq a$ implies $g(x) \geq g(a) \geq 0$. Then

$$
g(a) P(X \geq a) \leq E(g(X)) .
$$

Similarly, suppose that $x \leq a$ implies $g(x) \geq g(a) \geq 0$. Then

$$
g(a) P(X \leq a) \leq E(g(X)) .
$$

Proof. The first inequality follows from

$$
g(a) P(X \geq a)=g(a) \int_{x \geq a} \mathrm{~d} F(x) \leq \int_{x \geq a} g(x) \mathrm{d} F(x) \leq E(g(X)) .
$$

The proof of the second inequality is analogous. 
If $g$ is positive and increasing, Equation (13) holds for any value of $a$. Note also that (13) implies the weaker inequality $g(a) P(X>a) \leq E(g(X))$. Similar remarks apply to Equation (14) for a positive and decreasing $g$ and for the inequality $g(a) P(X<a) \leq E(g(X))$.

The next result provides bounds on $E U(X)$ complementary to Jensen's inequality when $U$ has bounded support.

Theorem 12 Let $U$ be absolutely continuous with density function $u$ and $S_{U}=$ $[a, b]$.

i) If $U$ is convex, then

$$
E U(X) \leq E u(X)(b-a) .
$$

ii) If $U$ is concave, then

$$
E U(X) \geq 1-E u(X)(b-a) .
$$

Proof. When $U$ is convex, its density function $u$ is increasing. Hence, applying the first inequality in Lemma 11 ,

$$
P(X \geq V)=\int_{a}^{b} u(v) P(X \geq v) \mathrm{d} v \leq \int_{a}^{b} E u(X) \mathrm{d} v=E u(X)(b-a) .
$$

When $U$ is concave, the density $u$ is decreasing. The weaker form of the second inequality in Lemma 11 gives

$$
g(a)[1-P(X \geq a)]=g(a) P(X<a) \leq E g(X) ;
$$

that is, $g(a) P(X \geq a) \geq g(a)-E g(X)$. Thus, $P(X \geq V)=\int_{a}^{b} u(v) P(X \geq v) \mathrm{d} v \geq \int_{a}^{b}[u(v)-E u(X)] \mathrm{d} v=1-E u(X)(b-a)$.

An expected utility maximizer is risk-averse if and only if $U$ is concave. Assuming risk-aversion, Jensen's inequality implies $U(E(X)) \geq E U(X)$ for any integrable $X$. Hence, under the assumptions of Theorem 12, risk-aversion implies the following bounds on the expected utility of any integrable r.v. $X$ :

$$
1-E u(X)(b-a) \leq E U(X) \leq U(E(X)) .
$$

Similarly, risk-seeking preferences give

$$
U(E(X)) \leq E U(X) \leq E u(X)(b-a) .
$$

For instance, assume risk seeking preferences with $U(x)=x^{2}$ on the support $[0,1]$. If the r.v. $X$ has c.d.f. $F(x)=\sqrt{x}$ on the same support, then

$$
U(E(X))=\frac{1}{9} \leq E U(X)=\frac{1}{5} \leq E u(X)(b-a)=\frac{2}{3} .
$$


The inequalities in Theorem 12 are sharp. For instance, assume that $V$ is uniformly distributed on $[0,1]$ and that $X$ is degenerate on $x=1$. Then $P(X \geq$ $V)=1=E u(X)(b-a)$ and (15) holds with equality. The same is true for (16) if we choose $X$ to be degenerate on $x=0$.

More interestingly, the bounds in Theorem 12 are (asymptotically) sharp for nondegenerate distributions. For instance, take the (convex) power utility function $U(x)=x^{\alpha}$ (with $\alpha \geq 1$ ) on the support [0,1]. For $k \in(0,1 / 2)$, assume that the r.v. $X$ takes values $k$ and $1-k$ with probability $1 / 2$. Then

$$
0 \leq E U(X)=\frac{1}{2}\left[k^{\alpha}+(1-k)^{\alpha}\right] \leq \frac{\alpha}{2}\left[k^{\alpha-1}+(1-k)^{\alpha-1}\right]=E u(X)(b-a) .
$$

For $\alpha \rightarrow+\infty$, the right-hand side tends to 0 and the upper bound becomes asymptotically sharp. Analogous results hold for the second inequality in Theorem 12.

The dual of Theorem 12 is the following.

Theorem 13 Let $X \sim F$ have an absolutely continuous c.d.f. with density function $f$ and bounded support $S_{F}=[a, b]$. If $F$ is convex, then $E U(X) \geq$ $1-E f(V)(b-a)$. If $F$ is concave, then $E U(X) \leq E f(V)(b-a)$.

\section{Moment generating functions}

Our last result relies on the moment generating functions. ${ }^{1}$ Since one is unlikely to know the moment generating functions better than the underlying c.d.f.'s, its interest is mostly theoretical.

Given a r.v. $X$, let $M_{X}(t)=E\left(e^{t X}\right)$ be its moment generating function (m.g.f.) and $D_{X}$ the domain over which $M_{X}(t)$ is defined. Given two stochastically independent r.v.'s $X \sim F$ and $V \sim U$, define the r.v. $Y=X-V$ and let $H$ be the c.d.f. of $Y$. For all positive $t$ in $D_{X} \cap D_{V}$, we have

$$
P(X \geq V)=P(Y \geq 0)=\int_{0}^{+\infty} \mathrm{d} H(y) \leq E\left(e^{t Y}\right)=M_{X}(t) \cdot M_{V}(-t) .
$$

The following result follows immediately.

Theorem 14 Suppose that the m.g.f.'s of $X$ and $V$ exist. Define the set $D=$ $D_{X} \cap D_{V} \cap \mathbb{R}^{+}$. Then

$$
E U(X) \leq \inf _{t \in D} M_{X}(t) \cdot M_{V}(-t) .
$$

This bound is trivial when the minimizer for the right-hand side is $t=0$. However, if $E(X)<E(V)<+\infty$, the derivative of $M_{X}(t) \cdot M_{V}(-t)$ is strictly negative in $t=0$ and thus the right-hand side of (17) is strictly decreasing on some right neighborhood of $t=0$. Therefore, the bound is certainly not trivial whenever $E(X)<E(V)$.

\footnotetext{
1 This approach was kindly suggested to us by Yosi Rinott.
} 
We consider an example. Suppose that both $X$ and $V$ are exponentially distributed with parameters respectively $\alpha>\beta>0$. Then $E(X)=(1 / \alpha)<(1 / \beta)=$ $E(V)$. Moreover, their m.g.f.'s

$$
M_{X}(t)=\frac{\alpha}{\alpha-t} \quad \text { and } \quad M_{V}(t)=\frac{\beta}{\beta-t}
$$

are respectively defined for $t<\alpha$ and $t<\beta$. The minimum of $M_{X}(t) \cdot M_{V}(-t)$ over $D$ is attained at $t^{*}=(\alpha-\beta) / 2>0$, which gives $E U(X) \leq(4 \alpha \beta) /(\alpha+\beta)^{2}<$ 1.

\section{References}

Berhold, M.H.: The use of distribution functions to represent utility functions. Management Science 19, 825-829 (1973)

Billingsley, P.: Probability and measure, 2nd edn. New York Chichester: Wiley 1986

Birge, J., Teboulle, M.: Upper bounds on the expected value of a convex function using gradient and conjugate function information. Mathematics of Operations Research 14, 745-759 (1989)

Bordley, R.F., LiCalzi, M.:, Decision analysis using targets instead of utility functions. Decisions in Economics and Finance 23 (2000) (forthcoming)

Castagnoli, E., LiCalzi, M.: Expected utility without utility. Theory and Decision 41, 281-301 (1996)

Davidson, J.: Stochastic limit theory. Oxford: Oxford University Press 1994

DellaVigna, S.: Preferenze dipendenti dalla storia e dal contesto. Undergraduate Thesis, Milan, Bocconi University (1997)

Grandmont, J.-M.: Continuity properties of a von Neumann-Morgenstern utility. Journal of Economic Theory 4, 45-57 (1972)

Kahneman, D., Tversky, A.: Prospect theory: an analysis of decision under risk. Econometrica 47, 263-291 (1979)

Karlin, S., Novikoff, A.: Generalized convex inequalities. Pacific Journal of Mathematics 13, 12511279 (1963)

Nieddu, F.: La théorie du benchmarking: ordres stochastiques, limitations supérieures et inférieures. Mémoire de D.E.A., Ecole Normale Supérieure de Cachan (1997)

Shiryayev, A.N.: Probability. Berlin Heidelberg New York: Springer 1984

Vickson, R.G.: Stochastic orderings from partially known utility functions. Mathematics of Operations Research 2, 244-252 (1977)

Willassen, Y.: Expected utility, Chebichev bounds, mean-variance analysis Scandinavian Journal of Economics 83, 419-428 (1981)

Willassen, Y.: Best upper and lower Tchebycheff bounds on expected utility. Review of Economic Studies 57, 513-520 (1990)

Wrather, C., Yu, P.L.: Probability dominance in random outcomes. Journal of Optimization Theory and Applications 36, 315-334 (1982) 